PROCEEDINGS OF THE

AMERICAN MATHEMATICAL SOCIETY

Volume 137, Number 2, February 2009, Pages 745-750

S 0002-9939(08)09511-7

Article electronically published on August 25, 2008

\title{
A METRIC SPACE WITH THE HAVER PROPERTY WHOSE SQUARE FAILS THIS PROPERTY
}

\author{
ELŻBIETA POL AND ROMAN POL
}

(Communicated by Alexander N. Dranishnikov)

\begin{abstract}
Haver introduced the following property of metric spaces $(X, d)$ : for each sequence $\epsilon_{1}, \epsilon_{2}, \ldots$ of positive numbers there exist collections $\mathcal{V}_{1}, \mathcal{V}_{2}, \ldots$ of open subsets of $X$, the union $\bigcup_{i} \mathcal{V}_{i}$ of which covers $X$, such that the members of $\mathcal{V}_{i}$ are pairwise disjoint and every member of $\mathcal{V}_{i}$ has diameter less than $\epsilon_{i}$. We construct two separable complete metric spaces $\left(X_{0}, d_{0}\right),\left(X_{1}, d_{1}\right)$ with the Haver property such that $d_{0}, d_{1}$ generate the same topology on $X_{0} \cap X_{1} \neq \emptyset$, but $\left(X_{0} \cap X_{1}, \max \left(d_{0}, d_{1}\right)\right)$ fails this property. In particular, the square of a separable complete metric space with the Haver property may fail this property. Our results answer some questions posed by Babinkostova in 2007.
\end{abstract}

\section{INTRODUCTION}

A metric space $(X, d)$ has the Haver property if for each sequence $\epsilon_{1}, \epsilon_{2}, \ldots$ of positive numbers there exists a sequence $\mathcal{V}_{1}, \mathcal{V}_{2}, \ldots$ of collections of open subsets of $X$, the union $\bigcup_{i} \mathcal{V}_{i}$ of which covers $X$, such that, for $i=1,2, \ldots$, the members of $\mathcal{V}_{i}$ are pairwise disjoint and each member of $\mathcal{V}_{i}$ has diameter less than $\epsilon_{i}$; see $[6]$.

The property $C$ of a space $X$ means that for any sequence $\mathcal{U}_{1}, \mathcal{U}_{2}, \ldots$ of open covers of $X$ there exists a sequence $\mathcal{V}_{1}, \mathcal{V}_{2}, \ldots$ of collections of open subsets of $X$, the union $\bigcup_{i} \mathcal{V}_{i}$ of which covers $X$, such that, for $i=1,2, \ldots$, the members of $\mathcal{V}_{i}$ are pairwise disjoint and each member of $\mathcal{V}_{i}$ is contained in a member of $\mathcal{U}_{i}$; see [1] and 5 .

A metrizable space $X$ has the property $C$ if and only if for any metric $d$ on $X$ generating the topology, $(X, d)$ has the Haver property (see sec. 4 (D) and [2] for more information).

The aim of this note is the following theorem, where $\vee$ stands for the maximum of two functions considered on their common domain.

Theorem 1.1. There are separable complete metric spaces $\left(X_{0}, d_{0}\right),\left(X_{1}, d_{1}\right)$ with the property $C$ such that $d_{0}, d_{1}$ generate the same topology on $X_{0} \cap X_{1} \neq \emptyset$ and $\left(X_{0} \cap X_{1}, d_{0} \vee d_{1}\right)$ fails the Haver property.

This readily yields the following:

Corollary 1.2. There is a separable complete metric space with the property $C$ whose square fails the Haver property.

Received by the editors September 24, 2007, and, in revised form, January 25, 2008.

2000 Mathematics Subject Classification. Primary 54D20, 54F45, 54E50.

Key words and phrases. Haver property, property $C$, product spaces.

The first author was partially supported by MNiSW Grant No. N201 034 31/2717. 
Indeed, the free union $(X, d)$ of the metric spaces $\left(X_{0}, d_{0}\right)$ and $\left(X_{1}, d_{1}\right)$ from Theorem 1.1 has these properties, since the metric space $\left(X_{0} \cap X_{1}, d_{0} \vee d_{1}\right)$ embeds isometrically into the square of $(X, d)$ with the metric $\rho\left(\left(x_{1}, y_{1}\right),\left(x_{2}, y_{2}\right)\right)=$ $\max \left(d\left(x_{1}, x_{2}\right), d\left(y_{1}, y_{2}\right)\right)$.

A completely metrizable separable space with the property $C$ whose square fails this property was constructed by Jan van Mill and R. Pol [10], and this construction is a key element in our proof of Theorem 1.1.

Corollary 1.2 answers some questions asked by Liljana Babinkostova [2, Problems 1 and 2]; see also sec.4 (A).

Let us notice that by [2, Theorem 15], the product of a metric space with the Haver property and a countable-dimensional metric space (i.e., a countable union of zero-dimensional sets; see [5]) always has the Haver property, while the property $C$ may be lost upon multiplication by a zero-dimensional space; see [11].

\section{A construction by Jan van Mill and R. Pol}

Our proof of Theorem 1.1 is based on a construction from [10, which we shall use to the following effect.

Proposition 2.1. There exist a compact metrizable space $E$, a subspace $S \subset E$, $\sigma$-compact sets $H_{0}, H_{1}$ in $E \backslash S$ and a sequence $\left(A_{n}, B_{n}\right), n=1,2, \ldots$, of pairs of disjoint closed sets in $E$ such that

(1) $H_{0} \cap H_{1}=\emptyset$ and $E \backslash S=H_{0} \cup H_{1}$,

(2) if $T \subset S$ is uncountable-dimensional, then its closure $\bar{T}$ in $E$ intersects both $H_{0}$ and $H_{1}$,

(3) the sequence $\left(A_{n} \cap S, B_{n} \cap S\right), n=1,2, \ldots$, is essential in $S$.

The condition (3) means that whenever $L_{i}$ is a partition in $S$ between $A_{i} \cap S$ and $B_{i} \cap S$, then $\bigcap_{i} L_{i} \neq \emptyset$; see [5, Definition 6.1.1].

Let us explain how to derive this proposition from the results in [10. We shall call a compact space $L$ a Bing compactum if for any continua $L_{1}, L_{2}$ in $L$ with $L_{1} \cap L_{2} \neq \emptyset$, either $L_{1} \subset L_{2}$ or $L_{2} \subset L_{1}$; see 9 .

Let $\mathbb{K}$ be the Cantor set in $I=[0,1]$, let $I^{\mathbb{N}}$ be the Hilbert cube and let $C_{i}=$ $\left\{\left(x_{0}, x_{1}, \ldots\right): x_{i}=0\right\}, D_{i}=\left\{\left(x_{0}, x_{1}, \ldots\right): x_{i}=1\right\}$ be the opposite faces in $I^{\mathbb{N}}$. Let us fix a partition $L$ between $C_{0}$ and $D_{0}$ in $I^{\mathbb{N}}$ which is a Bing compactum (cf. [3] or [9, sec. 3.8]), and let us set $E=\mathbb{K} \times L, C_{n}^{*}=E \cap\left(\mathbb{K} \times C_{n}\right), D_{n}^{*}=E \cap\left(\mathbb{K} \times D_{n}\right)$. Let $p: E \rightarrow \mathbb{K}$ be the projection onto the first coordinate. Recall that for any sequence $L_{1}, L_{2}, \ldots$, where $L_{n}$ is a partition in $I \times I^{\mathbb{N}}$ between the faces $I \times C_{n}$ and $I \times D_{n}$, for $n=1,2, \ldots$, there is a continuum in $(I \times L) \cap \bigcap_{n=1}^{\infty} L_{n}$ joining the faces $\{0\} \times I^{\mathbb{N}}$ and $\{1\} \times I^{\mathbb{N}}$. Therefore, by Rubin, Schori and Walsh [13] (see also [9, Theorem 3.9.3]), there is a first Baire class function $f: \mathbb{K} \rightarrow E$ with $p \circ f(t)=t$, for $t \in \mathbb{K}$, such that for any sequence of partitions $L_{n}$ in $E$ between $C_{n}^{*}$ and $D_{n}^{*}$, the intersection $\bigcap_{n=1}^{\infty} L_{n}$ hits $S=f(\mathbb{K})$. Enlarging $C_{n}^{*}$ and $D_{n}^{*}$ to open sets $V_{n}$ and $W_{n}$ with disjoint closures, and taking $A_{n}=\overline{V_{n}}, B_{n}=\overline{W_{n}}$, we get (3), cf. [5, Lemma 1.2.9]. Now, the construction in [10, sec. 3], applied to $p: E \rightarrow \mathbb{K}$ and $f: \mathbb{K} \rightarrow E\left(\mathbb{K}=F\right.$, in the notation of [10]), provides $G_{\delta}$-sets $S_{j}, j=1,2, \ldots$, in $E$ such that $S_{j} \backslash S$ are pairwise disjoint, $S \cup \bigcup_{j \geq n} S_{j}$ is a $G_{\delta^{-}}$set for $n=1,2, \ldots$, and for any uncountable-dimensional $T \subset S$, the closure $\bar{T}$ in $E$ intersects all but finitely many $S_{j}$. 
The set $E \backslash S$ is $\sigma$-compact and $S_{i} \backslash S$ are pairwise disjoint $G_{\delta^{-}}$-sets in $E \backslash S$ such that each union $\bigcup_{j \geq n}\left(S_{j} \backslash S\right)$ is also $G_{\delta}$ in $E \backslash S$. Using the separation theorem [7, §30, VII], one can find pairwise disjoint $\sigma$-compact sets $F_{i} \supset S_{i} \backslash S$ with $\bigcup_{i} F_{i}=E \backslash S$, and we define $H_{\tau}=\bigcup\left\{F_{2 j+\tau}: j=0,1, \ldots\right\}, \tau=0,1$. This gives (1) and (2).

\section{Proof of Theorem 1.1}

We shall use the notation introduced in Proposition 2.1. Let us fix a metric $\rho$ on $E$ generating the topology, with the $\rho$-diameter of $E$ not greater than 1, and let $\delta_{1} \geq \delta_{2} \geq \ldots$ be a sequence of positive numbers such that the $\rho$-distance between $A_{n}$ and $B_{n}$ is not less than $\delta_{n}$. Let us write, for $\tau=0,1$,

(4) $H_{\tau}=\bigcup_{i} H_{\tau, i}$ with $H_{\tau, 1} \subset H_{\tau, 2} \subset \ldots$ compact.

We define continuous maps $p_{\tau}: H_{\tau} \rightarrow I^{\mathbb{N}}$ such that $p_{\tau}\left(H_{\tau}\right)$ is countable-dimensional and for any $i$ and $y \in p_{\tau}\left(H_{\tau, i} \backslash H_{\tau, i-1}\right)$, where $H_{\tau, 0}=\emptyset$, the fiber $p_{\tau}^{-1}(y)$ is disjoint from $H_{\tau, i-1}$ and has $\rho$-diameter not greater than $\delta_{i} \cdot 2^{-i}$; cf. [7, $\S 28$, IX], [8] and [5], Lemma 5.3.1]. Then the decomposition of $E$ into fibers $p_{\tau}^{-1}\left(p_{\tau}(x)\right)$, for $x \in H_{\tau}$, and singletons $\{x\}$, for $x \in S$, is upper-semicontinuous, and let us denote the quotient map by

(5) $\pi: E \rightarrow E^{*}, A^{*}=\pi(A)$, for $A \subset E$.

Since $\pi$ embeds $S$ homeomorphically into $E^{*}$, we shall identify $S$ with its image $S^{*}$ (notice also that $S=\pi^{-1}\left(S^{*}\right)$ ). We have

(6) $H_{0}^{*} \cap H_{1}^{*}=\emptyset$ and $E^{*} \backslash S=H_{0}^{*} \cup H_{1}^{*}$.

Let us define

(7) $X_{\tau}=S \cup H_{\tau}^{*}$, for $\tau=0,1$.

Since, by (6), $E^{*} \backslash X_{\tau}=H_{1-\tau}^{*}$, we infer that

(8) $X_{\tau}$ is a $G_{\delta^{-}}$set in $E^{*}$.

Claim A. $X_{\tau}$ has the property $C$.

To see this, let us first notice that

(9) if $T \subset S$ is closed in $X_{\tau}$, then $T$ is countable-dimensional.

Indeed, the closure of $T$ in $E^{*}$ is disjoint from $H_{\tau}^{*}$, and therefore the closure of $T$ in $E$ is disjoint from $H_{\tau}$. By (2) in Proposition 2.1, this shows that $T$ is countabledimensional.

Now, let us consider a sequence $\mathcal{U}_{1}, \mathcal{U}_{2}, \ldots$ of open covers of $X_{\tau}$. Since $X_{\tau} \backslash S$ is countable-dimensional, we have $X_{\tau} \backslash S=\bigcup_{n=1}^{\infty} Z_{n}$, where $Z_{n}$ is 0-dimensional. For $n=1,2, \ldots$, choose a family $\mathcal{V}_{2 n}$ of pairwise disjoint open subsets of $X_{\tau}$ refining $\mathcal{U}_{2 n}$ and covering $Z_{n}$ (cf. [5, hint to Problem 6.3.D(a)]). Then $L=X_{\tau} \backslash \bigcup_{n=1}^{\infty} \cup \mathcal{V}_{2 n}$ is a subset of $S$ which is closed in $X_{\tau}$, hence by (9), it is countable-dimensional. By the same argument, for every $n=1,2, \ldots$ there is a disjoint family $\mathcal{V}_{2 n+1}$ of open sets in $X_{\tau}$ refining $\mathcal{U}_{2 n+1}$ such that $L \subset \bigcup_{n=1}^{\infty} \cup \mathcal{V}_{2 n+1}$.

Claim B. For $\tau=0,1$, there is a complete metric $d_{\tau}$ on $X_{\tau}$ generating the topology such that for the metric $d_{0} \vee d_{1}$ on $S=X_{0} \cap X_{1}$, the $\left(d_{0} \vee d_{1}\right)$-distance between $A_{n}^{*} \cap S, B_{n}^{*} \cap S$ is positive, for $n=1,2, \ldots$.

To check this claim we begin with the observation that, from (4) and (5),

$$
A_{m}^{*} \cap B_{m}^{*} \subset H_{0, m}^{*} \cup H_{1, m}^{*}
$$


Indeed, if $i>m$, the $\rho$-distance between $A_{m}$ and $B_{m}$ is not less than $\delta_{m} \geq \delta_{i}$ and the fibers $p_{\tau}^{-1}\left(p_{\tau}(x)\right)$ with $x \in H_{\tau, i} \backslash H_{\tau, i-1}$ have diameters not greater than $\delta_{i} \cdot 2^{-i}$. In effect, no fiber $\pi^{-1}(y)$ with $y \notin H_{0, m}^{*} \cup H_{1, m}^{*}$ intersects both $A_{m}$ and $B_{m}$.

For each $m$, fix open sets $V_{0}(m), V_{1}(m)$ in $E^{*}$ such that

$$
H_{\tau, m}^{*} \subset V_{\tau}(m) \text { for } \tau=0,1 \text {, and } \overline{V_{0}(m)} \cap \overline{V_{1}(m)}=\emptyset,
$$

where the closures are considered in $E^{*}$. From (10) and (11) it follows that $A_{m}^{*} \cap$ $V_{1-\tau}(m) \cap X_{\tau}$ and $B_{m}^{*} \cap V_{1-\tau}(m) \cap X_{\tau}$ have disjoint closures in $X_{\tau}$; hence there exist continuous maps $\varphi_{m}^{\tau}: X_{\tau} \rightarrow[0,1]$ such that

(12) $\varphi_{m}^{\tau}(a)=0, \varphi_{m}^{\tau}(b)=1$, whenever $a \in A_{m}^{*} \cap V_{1-\tau}(m), b \in B_{m}^{*} \cap V_{1-\tau}(m)$.

Let $\rho^{*}$ be any metric on $E^{*}$ generating the topology and let $\sigma_{\tau}$ be a complete metric on $X_{\tau}$ generating the topology, cf. (8). We set, for $\tau=0,1$ and $x, y \in X_{\tau}$,

$$
d_{\tau}(x, y)=\rho^{*}(x, y)+\sigma_{\tau}(x, y)+\sum_{m=1}^{\infty} 2^{-m}\left|\varphi_{m}^{\tau}(x)-\varphi_{m}^{\tau}(y)\right| .
$$

Then $d_{\tau}$ is a complete metric generating the topology on $X_{\tau}$. We will show that $d_{0}$ and $d_{1}$ satisfy the conditions of Claim B.

Let us fix $m$. For $\tau=0,1$ take an open set $W_{\tau}(m)$ in $E^{*}$ such that

$$
H_{\tau, m}^{*} \subset W_{\tau}(m) \subset \overline{W_{\tau}(m)} \subset V_{\tau}(m),
$$

cf. (11). Let $\eta_{\tau}$ be the $\rho^{*}$-distance between the sets $\overline{W_{\tau}(m)}$ and $E^{*} \backslash V_{\tau}(m)$ in $E^{*}$ and let $\eta>0$ be the $\rho^{*}$-distance between the sets $A_{m}^{*} \backslash\left(W_{0}(m) \cup W_{1}(m)\right)$ and $B_{m}^{*} \backslash\left(W_{0}(m) \cup W_{1}(m)\right)$ in $E^{*}$. We shall check that setting $\epsilon_{m}=\min \left\{\eta_{0}, \eta_{1}, \eta, 2^{-m}\right\}$, we have

(15) $\left(d_{0} \vee d_{1}\right)(a, b) \geq \epsilon_{m}$, whenever $a \in A_{m}^{*} \cap S, b \in B_{m}^{*} \cap S$.

To prove (15), take $a \in A_{m}^{*} \cap S, b \in B_{m}^{*} \cap S$. If both $a, b$ are outside of $W_{0}(m) \cup$ $W_{1}(m)$, then $\left(d_{0} \vee d_{1}\right)(a, b)>\rho^{*}(a, b) \geq \eta$. Suppose now that $a \in W_{0}(m)$. If $b \notin V_{0}(m)$, then $\left(d_{0} \vee d_{1}\right)(a, b) \geq \rho^{*}(a, b) \geq \eta_{0}$. If $a, b \in V_{0}(m)$, then by (12), $\varphi_{m}^{1}(a)=0, \varphi_{m}^{1}(b)=1$, and hence $\left(d_{0} \vee d_{1}\right)(a, b) \geq 2^{-m}$, cf. (13). If $a \in W_{1}(m)$, we proceed similarly, with the index 0 replaced by 1 , and we can also replace $a$ by $b$ in this reasoning. In any case, $\left(d_{0} \vee d_{1}\right)(a, b) \geq \epsilon_{m}$, which justifies (15) and completes the proof of Claim B.

Claim C. The space $\left(X_{0} \cap X_{2}, d_{0} \vee d_{1}\right)$ fails the Haver property, where $d_{\tau}, \tau=0,1$, are as in Claim B.

Indeed, let $\epsilon_{m}>0$ be the $\left(d_{0} \vee d_{1}\right)$-distance in $S=X_{0} \cap X_{1}$ between the sets $A_{m}^{*} \cap S$ and $B_{m}^{*} \cap S$, for $m=1,2, \ldots$. Since $A_{m}^{*} \cap S=A_{m} \cap S$ and $B_{m}^{*} \cap S=B_{m} \cap S$, the sequence $\left(A_{m}^{*} \cap S, B_{m}^{*} \cap S\right)$ is essential in $S$, by Proposition 2.1 (3), and it follows that there are no disjoint open collections $\mathcal{V}_{1}, \mathcal{V}_{2}, \ldots$ in $S$ such that the union $\bigcup_{m} \mathcal{V}_{m}$ covers $S$ and every member of $\mathcal{V}_{m}$ has $\left(d_{0} \vee d_{1}\right)$-diameter less than $\epsilon_{m}$; cf. sec. 4 (C).

\section{Comments}

(A) There are two classical covering counterparts to $\sigma$-compactness: the Hurewicz property and the weaker Menger property; see [2]. Babinkostova 2] showed that the Hurewicz property of a metric space with the Haver property yields the property $C$ of this space and guarantees the Haver property of its square. 
One can show that under Martin's Axiom, if the Hurewicz property is replaced here by the Menger property, the first statement is no longer true, and the second one fails even if we assume that the space has property $C$ and its square has the Menger property; see [12] (this answers Problem 4 from [2] and shows that the answers to Problems 1 and 2 in [2] are negative, even if we assume the Menger property of the space).

(B) Corollary 1.2 shows that the statement of Theorem 16 in [2] is incorrect.

(C) The Haver property of $(X, d)$ yields the following: for any sequence $\left(A_{n}, B_{n}\right)$, $n=1,2, \ldots$, of pairs of closed sets such that the $d$-distance between $A_{n}$ and $B_{n}$ is not less than $\epsilon_{n}$ for some $\epsilon_{n}>0$, there are partitions $L_{n}$ between $A_{n}$ and $B_{n}$ in $X$ with $\bigcap_{n} L_{n}=\emptyset$, cf. [1]. Indeed, let $\mathcal{V}_{n}, n=1,2, \ldots$, be the collections given by the Haver property for the sequence $\epsilon_{1}, \epsilon_{2}, \ldots$ There are closed sets $F_{n} \subset \bigcup \mathcal{V}_{n}$ with $\bigcup_{n} F_{n}=X$, and the traces of the members of $\mathcal{V}_{n}$ on $F_{n}$ form a discrete collection $\mathcal{F}_{n}$ of closed sets with diameters less than $\epsilon_{n}$. Consider $A_{n}^{*}=A_{n} \cup \bigcup\left\{F \in \mathcal{F}_{n}\right.$ : $\left.F \cap A_{n} \neq \emptyset\right\}$ and $B_{n}^{*}=B_{n} \cup \bigcup\left\{F \in \mathcal{F}_{n}: F \cap A_{n}=\emptyset\right\}$, and let $L_{n}$ be any partition in $X$ between the closed disjoint sets $A_{n}^{*}$ and $B_{n}^{*}$. Then $\bigcap_{n} L_{n}=\emptyset$.

(D) Let $X$ be a metrizable space without property $C$ and let $\mathcal{U}_{1}, \mathcal{U}_{2}, \ldots$ be a sequence of open covers of $X$ witnessing the failure of this property. Let $d$ be a metric generating the topology of $X$ such that for each $n$, the family of balls $\mathcal{W}_{n}=\left\{B_{d}(x, 1 / n): x \in X\right\}$ refines $\mathcal{U}_{n}$, cf. [4, IX.9.4]. Then $(X, d)$ fails the Haver property. Indeed, there are no disjoint open collections $\mathcal{V}_{1}, \mathcal{V}_{2}, \ldots$ such that the union $\bigcup_{n} \mathcal{V}_{n}$ covers $X$ and every member of $\mathcal{V}_{n}$ has $d$-diameter less than $\frac{1}{n}$, as each such $\mathcal{V}_{n}$ would refine $\mathcal{W}_{n}$, and hence also $\mathcal{U}_{n}$.

\section{REFERENCES}

1. D. F. Addis and J. H. Gresham, A class of infinite dimensional spaces. Part I: Dimension theory and Alexandroff's problem, Fund. Math. 101 (3) (1978), 195-205. MR0521122 (80b:54041)

2. L. Babinkostova, When does the Haver property imply selective screenability?, Top. Appl. 154 (2007), 1971-1979. MR2319269

3. R. H. Bing, Higher-dimensional hereditarily indecomposable continua, Trans. Amer. Math. Soc. 71 (1951), 267-273. MR0043452 (13:265c)

4. J. Dugundji, Topology, Allyn and Bacon, Boston, 1966. MR0193606 (33:1824)

5. R. Engelking, Theory of Dimensions, Finite and Infinite, Heldermann Verlag, Berlin, 1989. MR 1363947 (97j:54033)

6. W. E. Haver, A Covering Property for Metric Spaces, Lecture Notes in Mathematics, vol. 375, Springer, 1974, 108-113. MR0365504 (51:1756)

7. K. Kuratowski, Topology I, Academic Press and PWN, New York and London, 1966. MR0217751 (36:840)

8. A. Lelek, On the dimension of remainders in compactifications (Russian), Soviet Math. Dokl. 6 (1965), 136-140. MR0187197(32:4651)

9. J. van Mill, The Infinite-Dimensional Topology of Function Spaces, North-Holland, Amsterdam, 2001. MR1851014(2002h:57031)

10. J. van Mill and R. Pol, A complete C-space whose square is strongly infinite-dimensional, Israel Journ. Math. 154 (2006), 209-220. MR2254540 (2007h:54008)

11. E. Pol, A weakly infinite-dimensional space whose product with the irrationals is strongly infinite-dimensional, Proc. AMS 98 (1986), 349-352. MR0854045 (88a:54083) 
12. E. Pol and R. Pol, On metric spaces with the Haver property which are Menger spaces, preprint.

13. L. Rubin, R. M. Schori and J. J. Walsh, New dimension-theory techniques for constructing infinite-dimensional examples, General Top. Appl. 10 (1979), 93-102. MR0519716(80e:54049)

Institute of Mathematics, University of Warsaw, Banacha 2, 02-097 Warszawa, POLAND

E-mail address: pol@mimuw.edu.pl

Institute of Mathematics, University of Warsaw, Banacha 2, 02-097 WarszaWa, POLAND

E-mail address: pol@mimuw.edu.pl 